\title{
Cognitive tuning, encoding, and the attribution of causality
}

\author{
STEPHEN G. HARKINS \\ Northeastern University, Boston, Massachusetts 02115 \\ and \\ JOHN H. HARVEY, LOIS KEITHLY, and MARVINA RICH \\ Vanderbilt University, Nashville, Tennessee 37240
}

\begin{abstract}
Subjects were given a transmission or reception set before or after viewing a videotape of an event which involved an extreme outcome. The results indicated that transmitters made relatively extreme attributions to plausible causal agents when the set was given prior but not subsequent to viewing the event. The results also showed that subjects who had been given a transmission set before viewing the videotape exhibited relatively high recognition of aspects of the event they observed. It is suggested that the data provide support for an encoding interpretation of transmission-reception differences in the attribution of causality.
\end{abstract}

Zajonc (1960) defined cognitive tuning in terms of an individual's principal role in the communication process (i.e., whether a person's task was to transmit or to receive information). Tuning sets are thought to affect the flexibility of an individual's cognitive structure, which in turn may affect impressions formed about others. Individuals set to transmit information are hypothesized to have a relatively fixed and polarized cognitive structure which results in the exclusion of contradictory information. Receivers, on the other hand, should tend to have a more open and flexible cognitive structure, resulting in more openness to contradictory information.

Consistent with Zajonc's (1960) formulation, Cohen (1961) found that individuals expecting to transmit an impression were more prone to suppress contradictory information and exhibited more polarized impressions than did expectant receivers. Leventhal (1962) reported that expectant transmitters developed more simplified and unified impressions than did expectant receivers. Brock and Fromkin (1968) found that expectant transmitters chose to listen more to information supportive of their initial impressions of another person than did expectant receivers. Mazis (1973) extended the tuning formulation beyond person perception and found that expectant transmitters

This research was supported by a grant from the Vanderbilt Research Council to the second author and was conducted while the first author held a research associateship (funded by NSF Grant BNS-76-19629) at Ohio State University. We wish to extend our thanks to Richard E. Petty, Gary Wells, and David Wilson for their comments on an earlier draft of this manuscript. Request reprints from Stephen Harkins, 360 Huntington Avenue, Boston, Massachusetts 02115. were more receptive to familiar than to novel information concerning the attributes of automobiles.

Researchers in the cognitive tuning area have mainly been concerned with cognitive structure or "packaging" at the conceptual level and at the empirical level have used an impression formation paradigm; they have tested either the way in which the information is packaged (Leventhal, 1962; Zajonc, 1960) or, once packaged, the type of additional information that will be incorporated (Brock \& Fromkin, 1968; Cohen, 1961; Mazis, 1973).

Harvey, Harkins, and Kagehiro (1976) extended the tuning set analysis to interpretive activities by manipulating tuning set and observing the effects on the attribution of causality. Subjects who had been given either a transmission or a reception set watched a videotape of a staged learning experiment in which a "learner" was shocked by a "teacher" when he made errors on a paired associate learning task. In one condition, these shocks resulted in the learner's exhibiting an extreme amount of psychological distress by the end of the experiment, as evidenced by the reading on a psychological distress meter visible to the subjects and to the teacher. In a second condition, the meter reading only reached a moderate level. When asked to account for the learner's reactions to the shocks, transmitters viewing the extreme outcome ascribed higher levels of causality to the teacher and the learner than did receivers, while there were no transmission-reception differences in attribution for viewers of the moderate outcome tape. Harvey et al. (1976) suggested that extreme outcomes (i.e., the learner's extreme reaction) represent eventualities that are not anticipated and that this may have stimulated expectant transmitters to develop a more definitive 
interpretation of the event, resulting in higher attributions of causality to plausible factors in the situation. In a second experiment designed to test this interpretation, Harvey et al. directly manipulated violation of expectancy by varying whether the teacher's act of shocking the leamer was discrepant with information about the teacher's attitude toward causing a person distress in the interests of advancing psychological knowledge. Consistent with the previous study, when a violation of expectancy occurred, the transmitter attributed higher levels of causality to the teacher, the only plausible cause in this case. Overall, these results suggest that tuning set affects not only organization and structure, but also interpretive activity-in this case, causal attributions.

Researchers concerned with cognitive tuning, whether interested in structure or interpretation, have been insensitive to the possible importance of the locus of the administration of the tuning sets relative to presentation of the stimulus materials. If, for example, transmission-reception differences were obtained only when these sets were given prior to exposure to the stimulus materials, this would suggest that tuning set affects the manner in which the materials were viewed and encoded. Conversely, if transmissionreception differences were obtained when the tuning manipulation was given subsequent to exposure to the stimulus materials, it would suggest that tuning set did not result in the materials' being encoded in some particular way, since the crucial aspects of the material were retrievable. The purpose of the present research was to determine whether the attributional differences obtained as a function of tuning set in the Harvey et al. (1976) study were mediated by the manner in which the event was observed and encoded or retrieved and reconstructed. In that study, transmission-reception differences in attribution were obtained when the tuning set was given prior to the viewing of an event with an extreme outcome. If these differences were to be obtained only when the set is given prior to the viewing of the videotape, encoding processes would be implicated. If subjects who are given the sets subsequent to viewing the tape were to duplicate the results of the pretape subjects, the way the tape is viewed would seem to be relatively unimportant in mediating interpretations of the event; rather, in this case, the data would suggest that retrieval and reconstruction of aspects of the event play an important role in the mediational process.

In the present study, memory data (recognition of the correct response from the paired associate learning task viewed in the tape) were collected in an attempt to examine the processes which mediate the effects of tuning set on attributions. For example, if recognition scores were relatively higher when the tuning set is given prior to the viewing of the event, and if transmitterreceiver attribution differences were found in this situation, then the basis for inferring an active encoding process would be strengthened.

\section{METHOD}

\section{Subjects}

The subjects were 50 college students, 25 male and 25 female, who participated in order to satisfy an introductory psychology course requirement at Vanderbilt University. Ten subjects were randomly assigned to each experimental condition.

\section{Procedure}

Each of the subjects was told that the study concerned the manner in which people observe and interpret events and that their task was to carefully observe a videotape of an actual experiment concerning learning and psychological distress which had been recently conducted.

The videotape consisted of an 8.5 -min staged verbal learning experiment. In the opening scene, the male experimenter met two male subjects. The word-association task was explained, and a drawing was held to assign randomly the two subjects to the roles of teacher and learner. The subject who was assigned the role of teacher was asked to await further instruction while the subject assigned the learner's role was escorted to another room.

In the second scene, the experimenter explained that the learner's task was to learn 15 word pairs. During the learning phase, the teacher's assignment was to read the list twice over an intercom system. During test trials, the teacher would supply the first word of each pair and the learner would reply with the correct paired associate. Incorrect responses would result in the administration of a $.5-\mathrm{sec}$ shock to the learner by the teacher. The experimenter then attached the bogus electrodes to the learner's wrist and finger and left the learner's room.

In the third scene, the teacher was shown in side view seated at a table with a microphone, recording sheets, and a distress meter at a camera range of approximately $10 \mathrm{ft}(3.048 \mathrm{~m})$. In reply to the experimenter's question, the teacher indicated that he had overheard all of the instructions given to the learner on the open intercom. The experimenter switched off the intercom and explained the following additional aspect of the experiment, of which the learner was unaware: The electrodes attached to the learner's wrist and finger also were connected to a galvanic skin response meter that indicated the degree of psychological distress experienced by the learner during the experiment. It was explained to the teacher that another purpose of the experiment was to examine the relationship between the learner's performance on the task and the amount of distress he experienced, as indicated by the meter. Part of the teacher's task was to record the level of distress exhibited by the learner during the test trials. After the teacher indicated his understanding of the instructions, the experimenter positioned himself $3 \mathrm{ft}(91.84 \mathrm{~cm})$ to the teacher's left and asked the teacher to begin the learning trials.

After the teacher went through the list twice, the test trials were begun. At this point, an insert consisting of a close-up view of the psychological distress meter (a 15-point scale with 0 labeled as "low tension" and 15 labeled as "high tension") appeared in the lower right-hand corner on the television monitor in the subject's room. The learner answered incorrectly for 8 of the 15 pairs, receiving a shock from the teacher for each error. The distress meter indicated that gradually increasing levels of tension were experienced by the learner with each succeeding error and accompanying administration of shock with a mean level of distress of 14 .

Twenty of the subjects were given the cognitive tuning manipulation prior to viewing the tape. Ten of these subjects were told that a second focus of the research involved the ability of observers to transmit their descriptions and interpretations of the events that occurred on the tape to another person. After viewing the tape, they were told that they would be asked to provide a description and interpretation of the events to Mr. Elliott, another member of the research team (transmission 
set). The other 10 subjects were told that a second focus of the research involved the ability of observers to receive information about the event they observed from another person and to incorporate this information into their descriptions and interpretations of the event. Therefore, after viewing the tape they would be asked to listen to a description and interpretation of the event provided by Mr. Elliott (reception set). Twenty of the subjects, 10 in each of the tuning set conditions, were given the tuning set manipulation subsequent to viewing the tape. Ten additional subjects were given no set and constituted a control group; these subjects merely viewed the tape and then were given a postexperimental questionnaire.

After viewing the tape, the subjects were conducted to another room, in which a person other than Mr. Elliott administered a questionnaire. This aspect of the procedure was portrayed as involving a brief questionnaire about what they had seen, and it was expected that subjects in all conditions except those given no set would be anticipating reception or transmission of a description and interpretation of the event after responding to the questionnaire. Thus, the basic design was a 2 by $2+1$ with tuning set (transmission vs. reception) and locus (pretape vs. posttape) as crossed factors and a no-set control group. The major dependent measures included ratings on 20-point scales anchored on the low end by "not very much at all" and on the high end by "very much," indicating the extent to which the learner's psychological reaction was caused by (1) the teacher and his actions, (2) the experimenter and his actions, and (3) the learner's personality (high sensitivity to stressful circumstances).

Subjects also used 20-point scales to rate (1) the amount of time they spent thinking about developing an interpretation of what was happening as they viewed the tape, (2) the amount of time they spent thinking about hearing another person's interpretation of what was happening, (3) the amount of time they spent thinking about matters unrelated to the research, and (4) the amount of psychological distress shown by the learner. Finally, the subjects were given the stimulus word of each of the paired associates that was used on the videotape and were asked to select the correct response from four possibilities in a multiple choice format.

After completing the questionnaire, the subjects were queried about what they expected to occur next by an experimenter who was blind to conditions. All of the subjects indicated the appropriate expectation (control subjects indicated that they did not anticipate any further activities). The subjects were then given a thorough debriefing and the session was terminated.

Each of the measures was analyzed in a 2 by 2 analysis of variance with tuning set (transmission vs. reception) and locus (pretape vs. post tape) as between-subjects factors. The NeumanKeuls test (Kirk, 1968) was used for a posteriori mean comparisons within this 2 by 2 . Comparisons of the no-set control with the other means were made using the Dunnett procedure (Kirk, 1968). The error term used for the Dunnett was the within-cells variance from a one-way ANOVA with all five experimental conditions as levels. The means for the main dependent measures are presented in Table 1.

\section{RESULTS}

\section{Attribution to Teacher}

This analysis revealed a Tuning Set by Locus interaction $[F(1,36)=4.1, p<.05]$. Subjects in the pretape transmission condition rated the teacher's behavior as more the cause than did the transmitters in the posttape condition $(\mathrm{p}<.05)$ or the receivers in either the pre- or posttape conditions (ps<.05). No other mean comparisons relevant to this interaction were significant ( $p s>.20$ ). The no-set control differed only from the pretape transmission mean $(p<.05)$.

\section{Attribution to Learner}

A Tuning Set by Locus interaction was also obtained on this measure $[F(1,36)=8.5, p<.05]$. As with attribution to teacher, subjects in the pretape transmission condition made higher attributions to the learner's personality than did transmitters in the posttape condition $(\mathrm{p}<.05)$ or receivers in the pre- or posttape conditions (ps $<.05)$. No other mean comparisons were significant (ps $>.20$ ). A main effect for tuning set was also obtained $[F(1,36)=11.3$, $\mathrm{p}<.01]$. Transmitters made more extreme attributions $(M=12.3)$ than did receivers $(M=8.5)$; however, this difference must be interpreted in terms of the interaction, which indicates that the main effect was the result of the high causal attributions made by pretape transmitters. The no-set control differed only from the pretape transmission cell $(\mathrm{p}<.05)$.

\section{Attribution to Experimenter}

No significant effects were obtained for this dependent measure.

\section{Recognition Data}

The errors made by the subjects on the multiple choice test of the paired associates were summed and the analysis performed on these scores revealed a Tuning Set by Locus interaction $[F(1,36)=6.3, p<.05]$ and a main effect for locus $[F(1,36)=12.9, p<.01]$.

Table 1

Attributions of Causality and Recognition Scores as a Function of Experimental Condition

\begin{tabular}{|c|c|c|c|c|c|}
\hline \multirow[b]{2}{*}{ Attributions to: } & \multicolumn{2}{|c|}{ Transmitters } & \multicolumn{2}{|c|}{ Receivers } & \multirow{2}{*}{$\begin{array}{l}\text { No-Set } \\
\text { Control }\end{array}$} \\
\hline & Pretape & Posttape & Pretape & Posttape & \\
\hline Teacher & $14.4^{*}$ & $8.5 \dagger$ & $9.1 \dagger$ & $9.5 \dagger$ & $9.8 \dagger$ \\
\hline Learner & $14.5^{*}$ & $10.0 \dagger$ & $7.5 \dagger$ & $9.5 t$ & $9.2 \dagger$ \\
\hline Experimenter & $7.7 *$ & $5.1^{*}$ & $5.6^{*}$ & $8.1 *$ & $4.9^{*}$ \\
\hline Recognition Errors & $2.3^{*}$ & $6.3 \dagger$ & $5.2 \dagger$ & $5.9 \dagger$ & $5.7 \dagger$ \\
\hline
\end{tabular}

Note-Means sharing a common sign (* or t) are not significantly different at $p<.05$. Although Newman-Keuls was used to make comparisons within the 2 by 2 and the Dunnett was used to make comparisons with the no-set controls, common signs were used to describe the pattern of differences. 
Pretape transmitters made fewer errors than did subjects in each of the other cells ( $p$ < $<.05$ ), none of which differed significantly from the others (ps $>.20)$. Pretape subjects made fewer errors $(M=3.8)$ than posttape subjects $(M=6.1)$, an effect which must be interpreted in terms of the interaction. No-set control subjects made more errors than pretape transmitters $(p<.05)$, but did not differ from subjects in any of the other cells $(\mathrm{ps}>.20)$. These results are shown in Table 1 .

\section{Ancillary Measures}

No significant differences were obtained on the question asking the subjects to rate the amount of distress exhibited by the learner. The overall level of rated distress $(M=17.7)$ was approximately the same as that obtained in the Harvey et al. (1976) research $(M=17.6)$.

Analysis of the question asking how much time was spent thinking about developing an interpretation of what was happening as they viewed the tape revealed a Tuning Set by Locus interaction $[F(1,36)=14.5$, $\mathrm{p}<.0001]$. Pretape transmitters spent more time thinking about it than did subjects in comparable conditions ( $p s<.05)$. No other differences relevant to the interaction were significant $(\mathrm{ps}>.20)$. A main effect for locus was also obtained $[F(1,36)=10.8$, $\mathrm{p}<.01]$, which was the result of pretape subjects indicating that they thought more about developing an interpretation $(M=9.7)$ than did posttape subjects $(M=5.2)$. The pretape transmitters also spent more time thinking about developing an interpretation than did the no-set controls $(\mathrm{p}<.05)$.

For the question about how much time they spent thinking about hearing another person's interpretation of the event on the tape, pretape receivers reported spending more time than any other group, resulting in a Tuning Set by Locus interaction $[F(1,36)=14.6$, $p<.0001]$. Main effects for locus $[F(1,36)=27.6$, $p<.0001]$ and tuning set $[F(1,36)=16.6, p<.001]$ must be interpreted in terms of the interaction. The locus main effect showed that pretape subjects indicated that they thought more about hearing an interpretation $(M=10.0)$ than did posttape subjects $(M=3.8)$, and the tuning set main effect was the result of receivers' indicating more thinking about hearing $(M=9.3)$ than transmitters $(M=4.4)$. Each of these effects was obtained as the result of pretape receivers' spending more time thinking about hearing than subjects in other comparable cells. Subjects in the pretape receiver cell also indicated that they spent more time thinking about hearing than did subjects in the no-set control condition $(p<.05)$, which did not differ from any of the other cells $(p s>.20)$.

No significant differences were obtained on the question asking how much time was spent thinking about matters that did not relate at all to what was observed on the tape.

\section{DISCUSSION}

When the tuning sets were given to subjects prior to their viewing the tape, the pattern of results replicated the findings of Harvey et al. (1976). Thus, expectant transmitters viewing an event with an extreme outcome attributed relatively greater causality to the teacher and the learner, the more plausible causal agents in the situation. Also, as in that research, no differences were obtained for attributions to the experimenter, who merely read instructions and stayed in the background. However, when tuning set was administered subsequent to viewing the tape, no attributional differences were obtained. These data suggest that transmission set affects the manner in which the videotaped event is observed and encoded. Posttape transmitters apparently were unable to retrieve and reconstruct the event in the same way.

The finding that pretape transmitters spent more time developing an interpretation so that another person could hear it accommodates the interpretation that these subjects were more stimulated to develop a relatively definitive interpretation of the event. Although pretape receivers reported spending more time thinking about hearing another person's interpretation, this reported effort resulted in no attributional differences between these subjects and posttape receivers. Considering the results for the no-set control subjects, which did not differ from the results for the pre- and posttape receivers, it appears that the reception set results in a passive observational strategy with no strong impact on interpretive activity. Since more information may be obtained from the person from whom they will be receiving an interpretation, receivers may not feel a need to develop an unequivocal interpretation.

The pattern of results for the recognition data mirrored the attributional data. Pretape transmitters remembered the paired associates more accurately than did subjects in comparable conditions, although even these subjects incorrectly identified an average of only 5.8 of the associates. This evidence suggests that the pretape transmission set stimulated these subjects to observe the tape more closely than subjects who had been given a pretape reception set, no set, or posttape sets. Presumably, this closer observation of the event and the concomitant encoding process mediated high attributions of causality to plausible causal factors on the part of pretape transmitters.

The results obtained in this study suggest that the transmission set and its locus are most influential in the effect of tuning set on attributions. From the results obtained in this study, no distinctions can be made between the attributional tendencies of receivers and subjects with no set. It is possible that these variations elicit the same psychological process (openness to information or whatever), but the present 
data do not permit conclusions about this possibility. When given prior to the event, the transmission set appears to stimulate individuals to watch the event carefully and to try to generate a rather conclusive interpretation. This observational process is also implied by the data showing that subjects given the responsibility to transmit the information after the event appear to be unable to reconstruct the event in the same way. In the present line of reasoning, it is assumed that the unexpectedly severe reaction to the shocks on the part of the learner, in concert with the close observation, leads to the high attributions to the plausible causal agents.

A theoretically less interesting possible alternative interpretation is that the pretape transmission manipulation simply resulted in a more alert subject, and that this alone accounts for the results. However, in the first experiment of the Harvey et al. (1976) study, expectant transmitters who saw the moderate distress tape did not differ in their attributions from expectant receivers who saw the moderate or extreme distress tapes. Also, in their second experiment, expectant transmitters who saw a moderate distress version of the videotape and whose expectancies concerning the teacher's behavior were not violated did not differ in their attributions from receivers, regardless of violation of expectancy. In each of these experiments, transmission set alone did not lead to higher attributions of causality; in both cases either the direct or indirect (extreme distress) violation of expectancy was required. Transmissionreception in the present study was manipulated as it was in those two experiments. All of the subjects in the three experiments were asked to watch the tape very closely. Further, regardless of this series of experiments, it is not clear why pretape receivers would not be expected to be just as alert as pretape transmitters. Thus, it seems unlikely that the pretape transmission manipulation simply enhanced the alertness of these subjects, since this manipulation alone was not sufficient to obtain differences in previous research (Harvey et.al., 1976).

It could also be argued that the effects obtained in the present research were the result of the fact that the information was to be transmitted to a member of the research team, who could evaluate the correctness of the subjects' responses. In the first experiment of the Harvey et al. (1976) study, the expertise of the recipient/source on the research team was manipulated. The person was described as either a clinical psychologist experienced in the analysis of interpersonal events or a college sophomore who was considering clinical psychology as an academic major. The attributional pattern was the same for each potential recipient/source, which suggests that it is unlikely that concern over the status of the recipient/source led to the pattern of results obtained in the present research.

It is unclear whether similar results would have been obtained if the presumed structural effects of tuning sets had been examined. In the first experiment reported by Zajonc (1960), he manipulated tuning set subsequent to the subjects' exposure to the stimulus materials, while in his second experiment he reversed the order. The fact that similar results were obtained suggests that the dependent measures designed to probe structural aspects were left unaffected by the locus of the manipulations. However, locus was not systematically manipulated in these experiments. Thus, the effects of the timing of tuning set on dependent variables relevant to structure cannot be clearly assessed.

A major contribution of the present work would seem to be its explicit focus on the locus variable and on how it interacts with the nature of the tuning set to affect attributions. This work again shows that cognitive set is an independent variable which is meaningfully related to interpretive activity.

\section{REFERENCES}

Brock, T. C.. \& Fromkin. H. L. Cognitive tuning set and behavioral receptivity to discrepant information. Journal of Personality. 1968, 36, 108-125.

Conen, A. R. Cognitive tuning as a factor affecting impression formation. Journal of Personality, 1961, 29, 235-245.

Harvey, J. H., Harkins, S. G., \& Kagehiro, D. K. Cognitive tuning and the attribution of causality. Journal of Personality and Social Psychology. 1976, 34, 708-715.

KIRK, R. E. Experimental design: Procedures for the behavioral sciences. Belmont: Brooks/Cole, 1968.

LEVENTHAL, $H$. The effects of set and discrepancy on impression change. Journal of Personality, 1962, 30, 1-15.

Mazis, M. B. Cognitive tuning and receptivity to novel information. Journal of Experimental Social Psychology, $1973,9,307-319$.

ZAJONC, R. The process of cognitive tuning in communications. Journal of Abnormal and Social Psychology, 1960, 61, 159-167.

(Received for publication February 8, 1977; revision accepted May 12, 1977.) 